\title{
ANALYTICAL STUDY OF THE EFFECT OF THE APPLICATION OF SMART BUILDINGS ON THE ENVIRONMENT OF ADMINISTRATIVE BUILDINGS AND THE RATE OF ENERGY CONSUMPTION WITHIN THEM
}

\author{
Ayman Hassan Ahmed Mahmoud ${ }^{1}$, Hussein Sabri Al-Shanwani ${ }^{2}$ \\ and Nermin Nabil Mohammed Attieh ${ }^{2}$ \\ ${ }^{1}$ Faculty of Engineering, Cairo University \\ ${ }^{2}$ Faculty of Engineering, Zagazig University,
}

\begin{abstract}
The evolution of modern technologies and integrated computer systems has made a major shift in the construction industry. This led to the emergence of "smart buildings" concept. The demand for this type of buildings is not only for the sake of luxury but also it is necessary to gain several advantages such as low energy and water consumption, decrease operating costs increasing productivity as well as providing the welfare of users; thus Smart buildings participate in positively affecting natural environment and human wellbeing. Administrative buildings' sector is one of the most energy consuming sectors in several countries; the research aims to explain the impact of smart systems on energy conservation strategies within the Administrative buildings while demonstrating the numerous environmental advantages of the smart architecture through the descriptive analysis of a number of smart administrative buildings in order to determine the reduction in energy consumption for these building.
\end{abstract}

Key words : Smart Buildings , Smart system , Integrated Systems , Energy consumption.

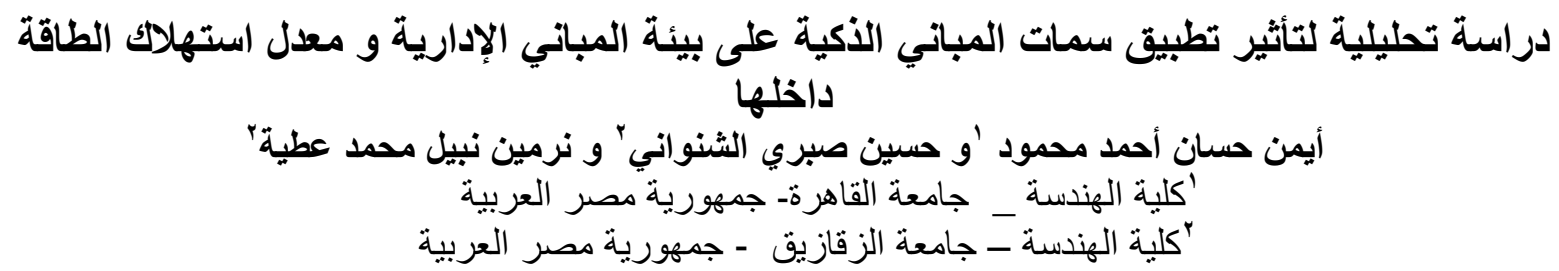

تطور التقنيات الحديثة و أنظمة الكمبيوتر المتكاملة أحدث تحول كبير فى صناعة البناء، مما أدى إلى ظهور مفهوم "

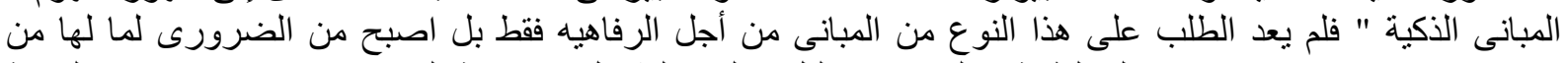

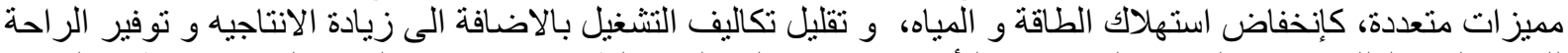

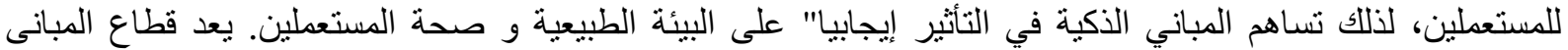

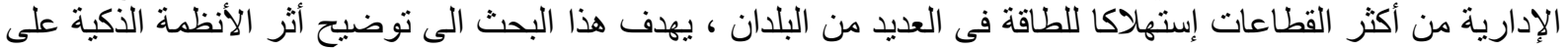

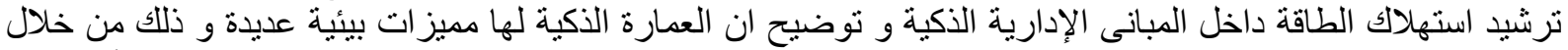

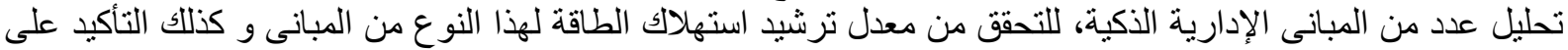

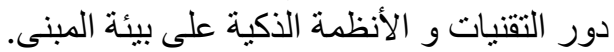
الكلمات الدالة : المبانى الأكية ، النظام الذكى ، الأنظمة المتكاملة ، استهلاك الطاقة . 


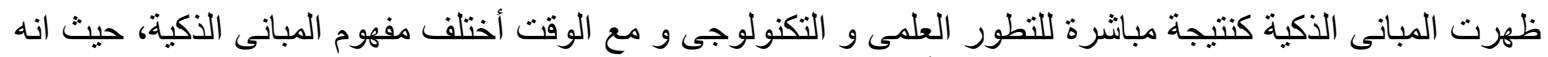

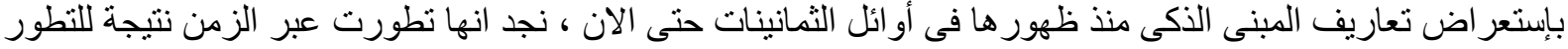

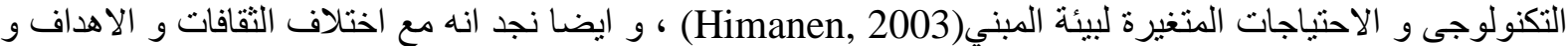

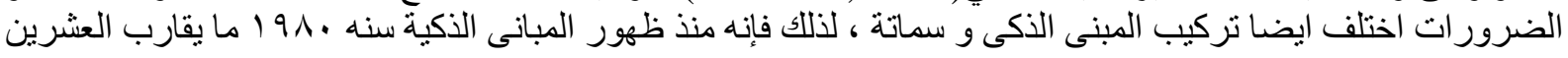
عاماو حتى الان من الصعب ان نجد تعريفا متفق عليه .

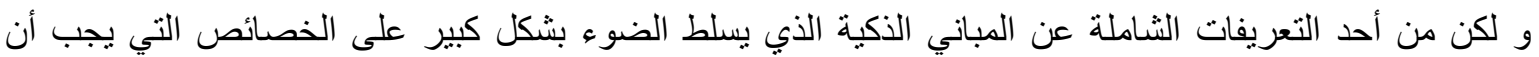

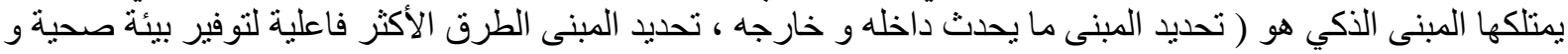

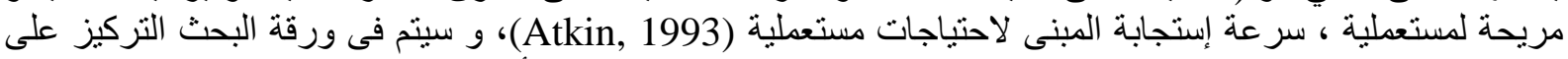
توضيح استر ايجيات ترشيد استهلاك الطاقة فى المبانى الإدارية الذية لئية بإستخدام الأنظمة الذكية . 1- 1 - المشكلة البحثية :

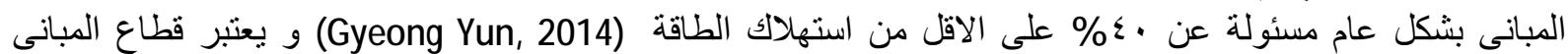

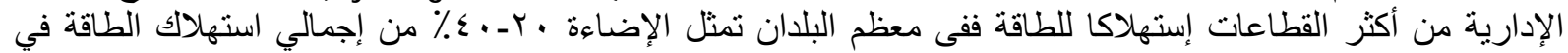

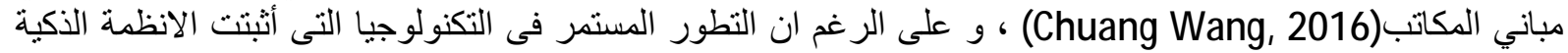

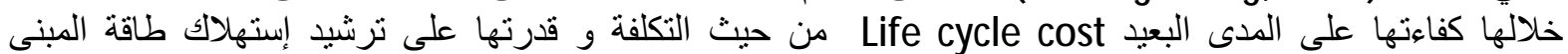

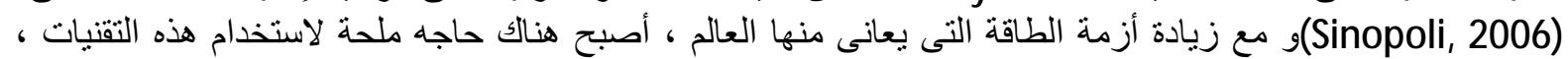

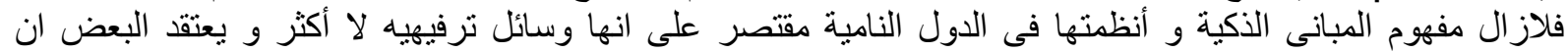

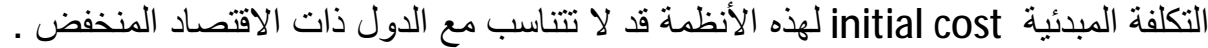

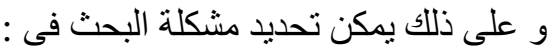
عدم وضوح الربط بين استخدام التقنيات و الأنظمة الذكية داخل المبانى و إمكانيتها فى الحفاظ على البيئة و ترشيد استهلاك

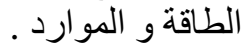

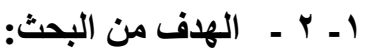

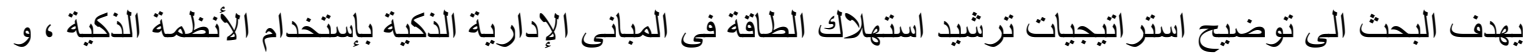

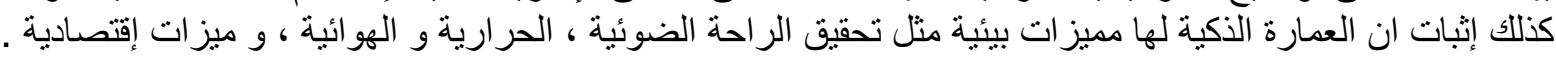

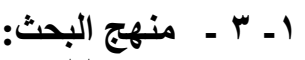
منهج وصفى تحليلي من اليحت: descriptive analysis من خلال :

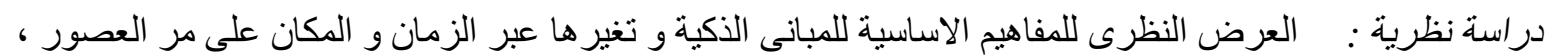

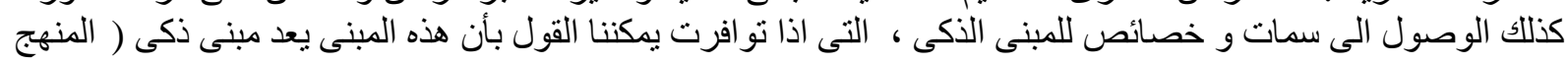
الاستقر ائى) .

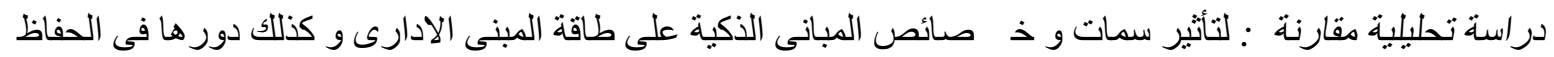

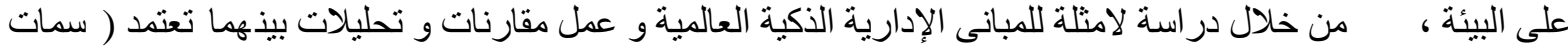

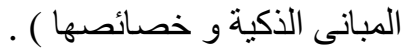

\section{1 - ـ ـ ـ نبذة تاريخية لنشأة و تطور فكرة المبانى الأكية:}

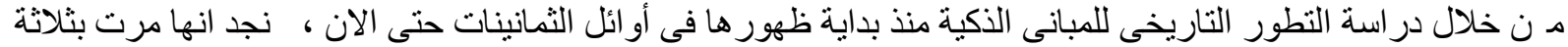

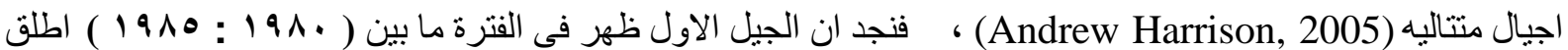

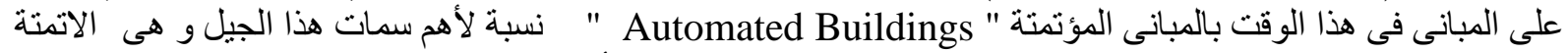
Automation بشرى و ذللك من خلال برمجه هذه الاله لتنفيذ العمل.

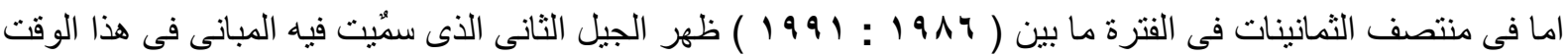

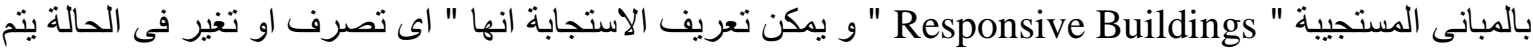

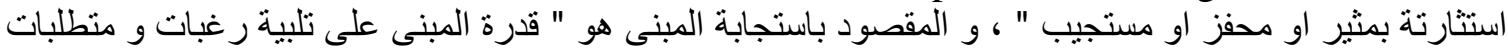
شاغلية و للتغير ات فى البيئة الداخلية و الخارجية للمبنى " .

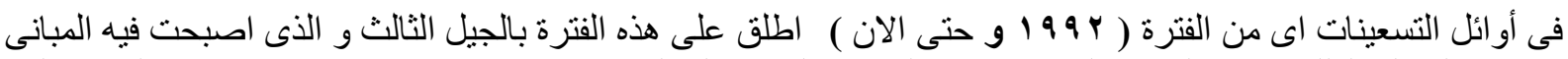

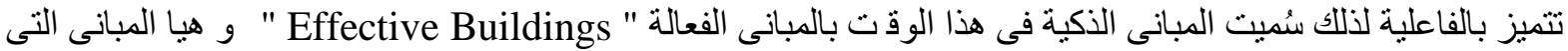
تمثل بيئة متجاوبة لتحقيق عملها (Elazm, 2017) و كان الهدف في هذا الوقت إيجاد بيئات عمر انية قادرة على تحمل 
مسئوليتها البيئية و تحقيق مبادئو مفاهيم الحفاظو الإستدامة ، من خلال الاعتماد على مصادر الطاقة المستدامة ، الكامنة ، المتجددة و غير ملوثة للبيئة .

مما سبق يمكننا تقسيم التطور التاريخى للعمارة الذكية منذ بداية ظهور ها فى الثمانينات و حتى الان الى ثلاثة حقبات

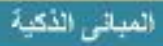
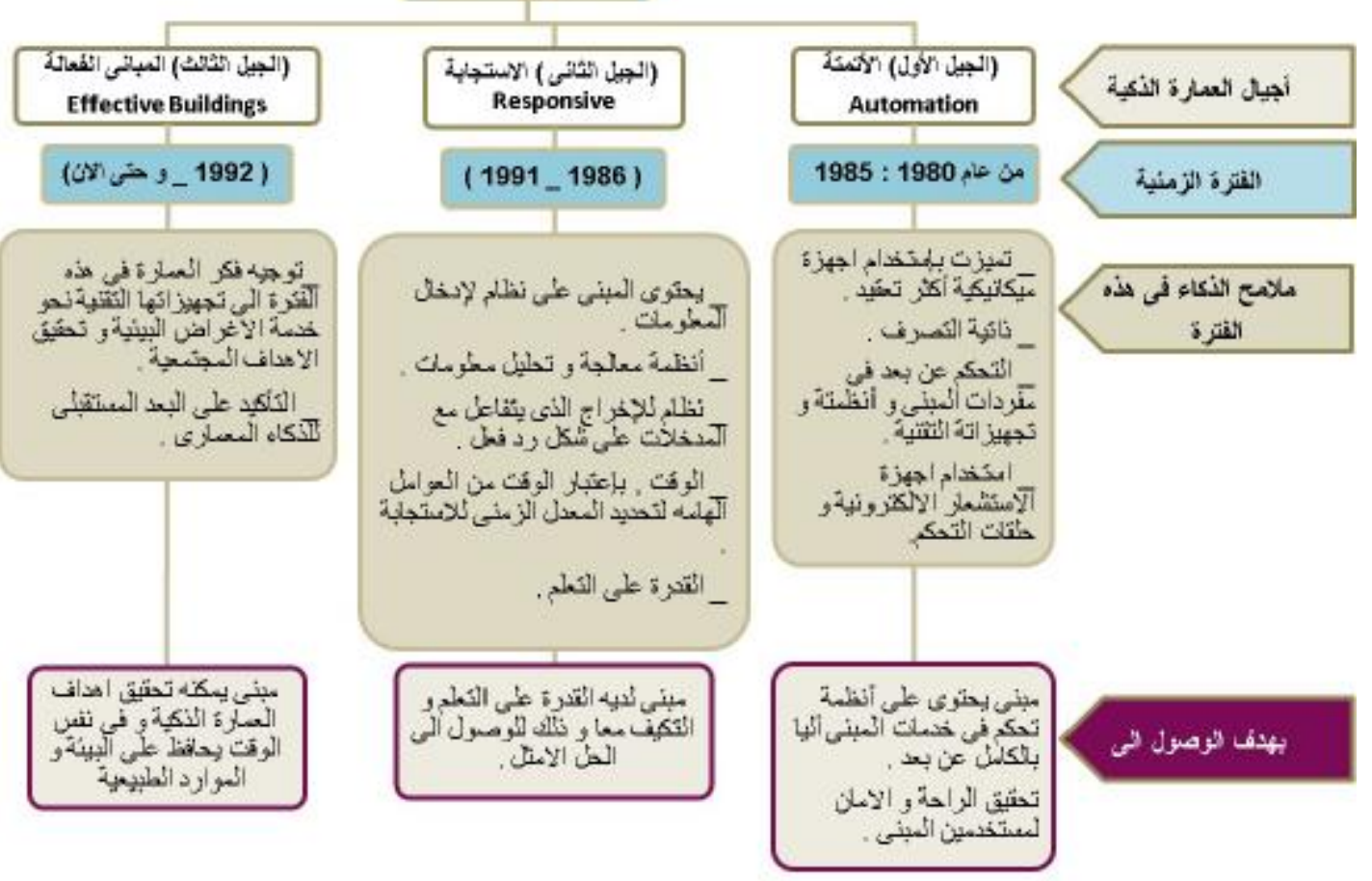

زمنية) (Andrew Harrison, 2005) أنظر شكل1.

شكل ا لتطور الأجيال الثلاثة للمبانى الذكية من خيث ملامح الذكاء لكل مرحلة و الهذف منها ( المصدر الباحث ) نقلا عن (Ahmed Reda, 2010)

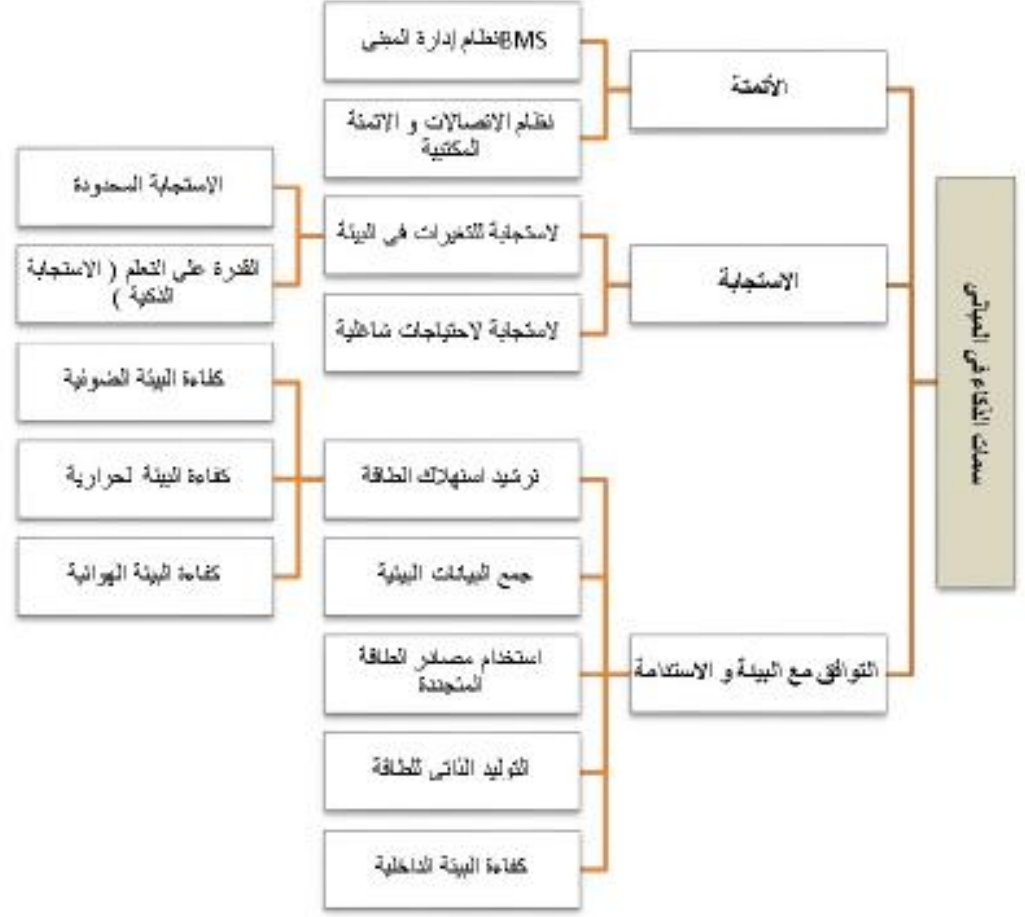

شكل r سمات الأكاء فى المبانى ( المصدر الباحث ) نقلا عن (Ahmed Reda, 2010) 


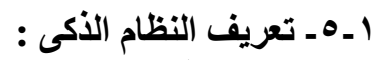

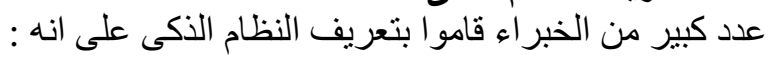

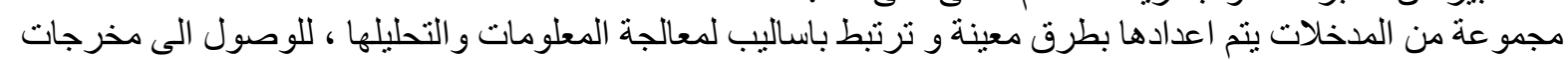

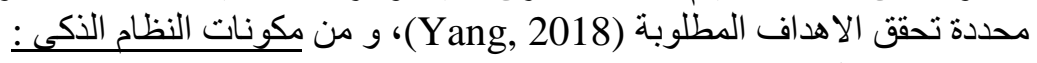
Information Processing Application and Analysis _ معالجة المعلومات و التحليل

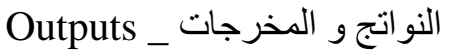

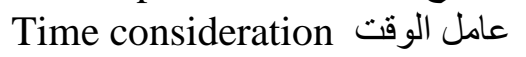

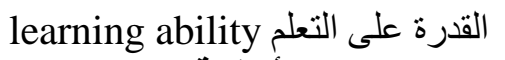

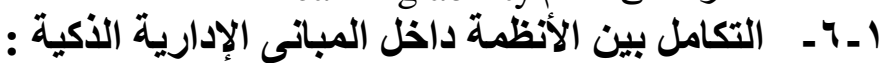

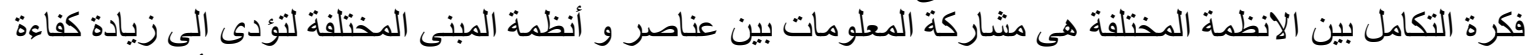

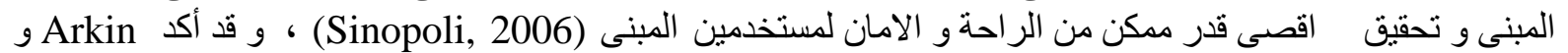

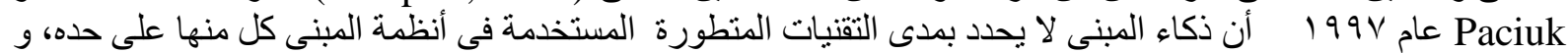

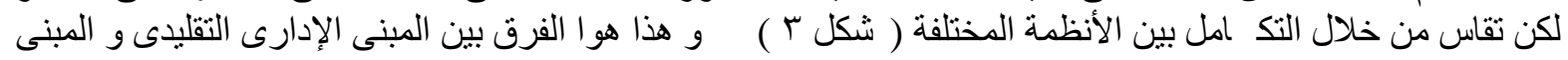
الإدارى الذكى الذى يوفر ل

(Montbonnot Saint Martin, 1999)
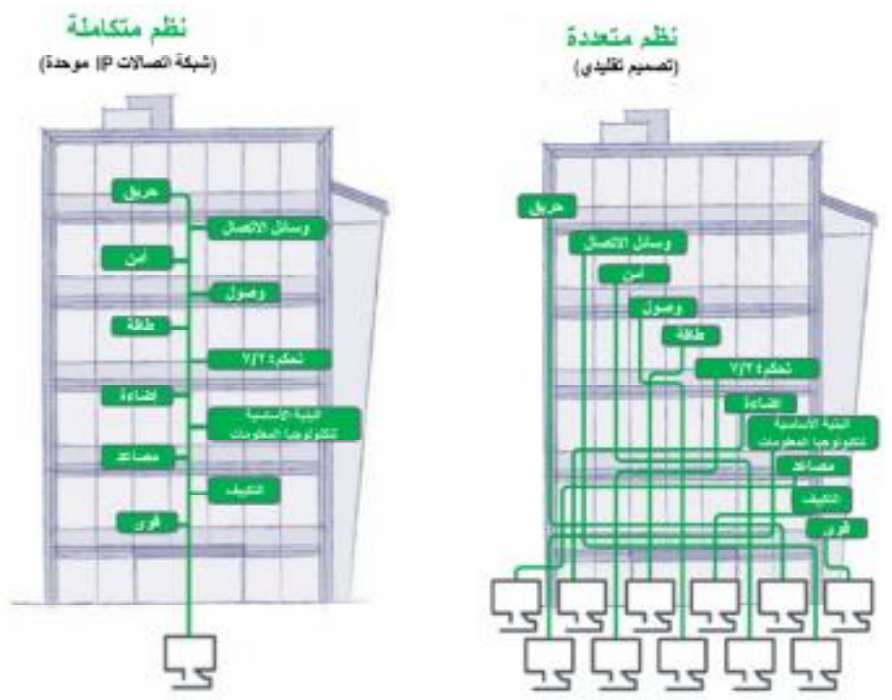

شكل ب يوضح الفرق بين عمل الأنظمة المتعددة بثكل منفصل و بثكل متكامل (Sotiris Papantoniou, 2017)

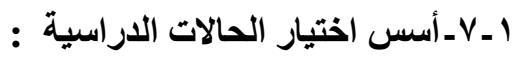

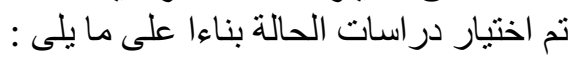

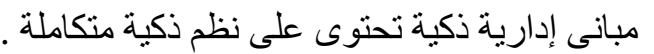

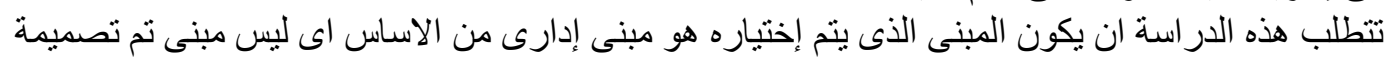

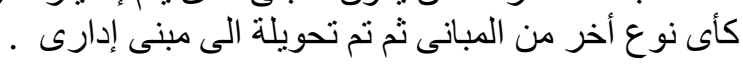

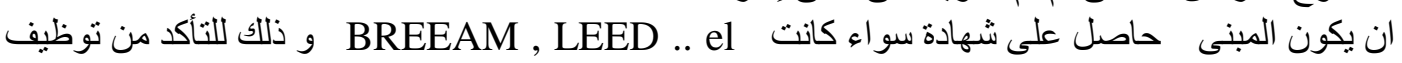
النظم الذكية فى ترشيد استهرلاك الطاقية 
جدول ا مقارنة للمبانى الإدارية الأكية من حيث سمات الذكاء فى المبنى

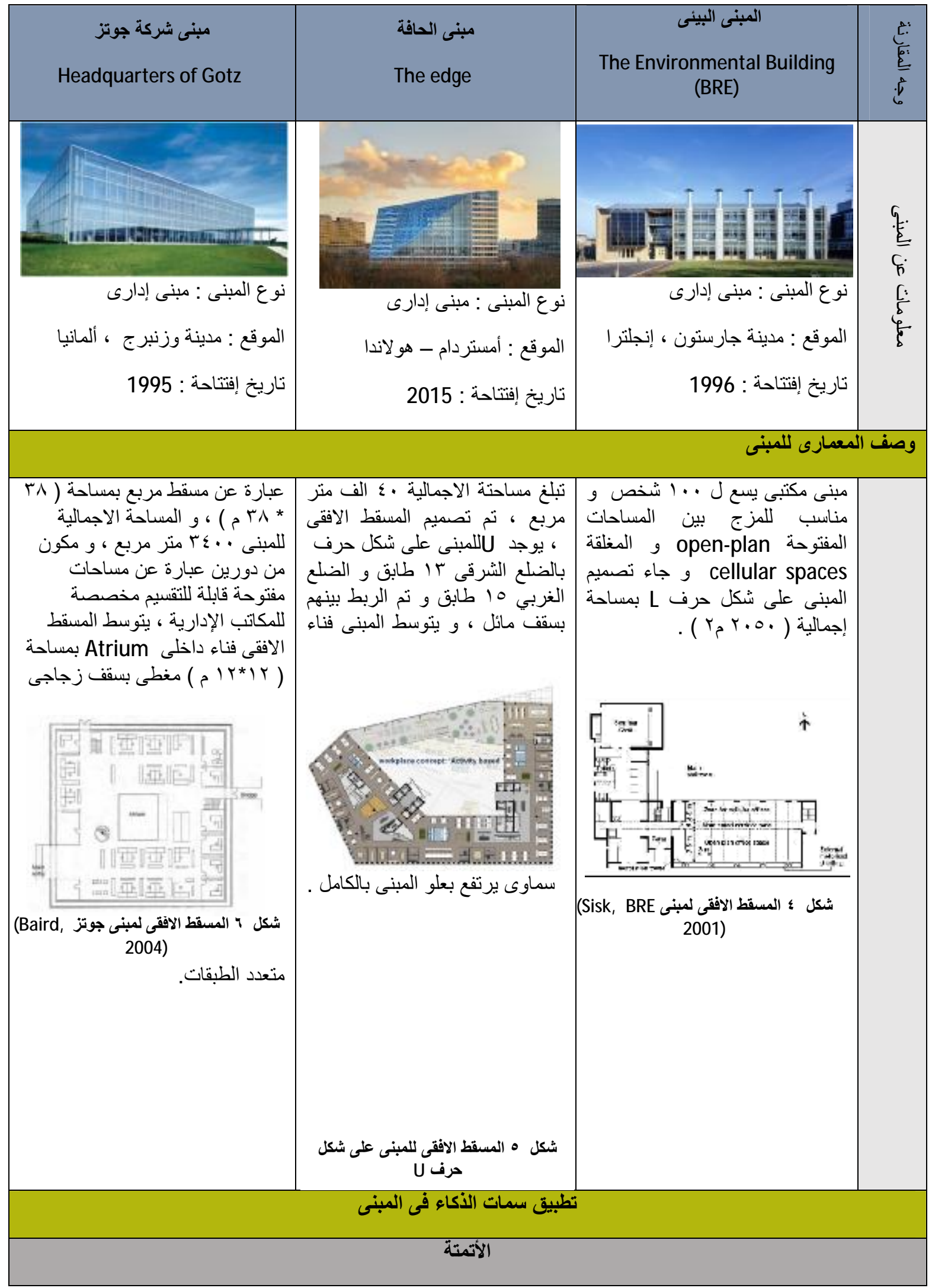




\begin{tabular}{|c|c|c|c|}
\hline 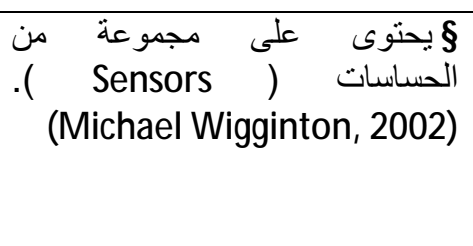 & 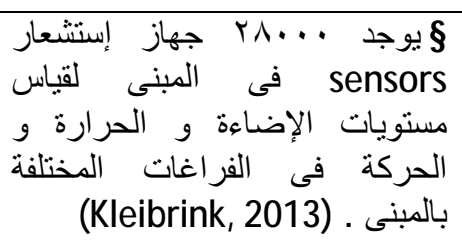 & 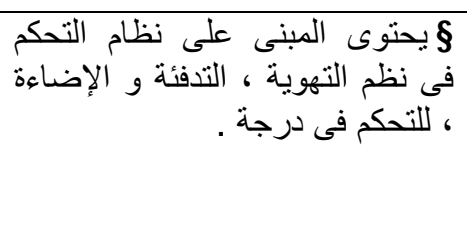 & $\underline{\underline{E}}$ \\
\hline 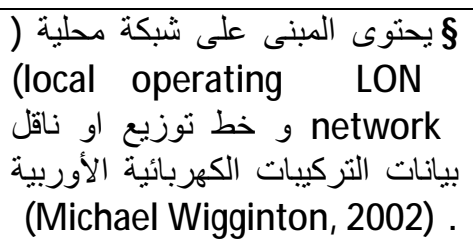 & 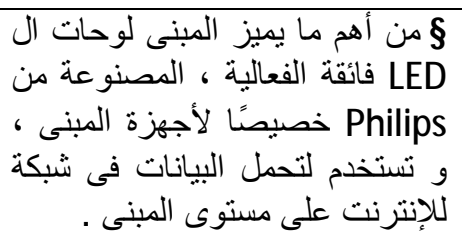 & 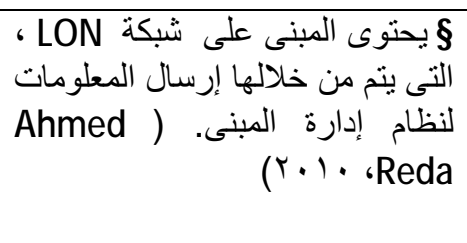 & $\begin{array}{l}2: \\
\vdots \\
\vdots \\
\vdots \\
\vdots \\
\vdots \\
\vdots \\
\vdots\end{array}$ \\
\hline $\begin{array}{l}\text { Headquarters of Gotz } \\
\text { Hركة جوتز }\end{array}$ & The edge & $\begin{array}{c}\text { المبنى البيئى } \\
\text { The Environmental } \\
\text { Building (BRE) }\end{array}$ & \\
\hline \multicolumn{4}{|c|}{ الإستجابة } \\
\hline 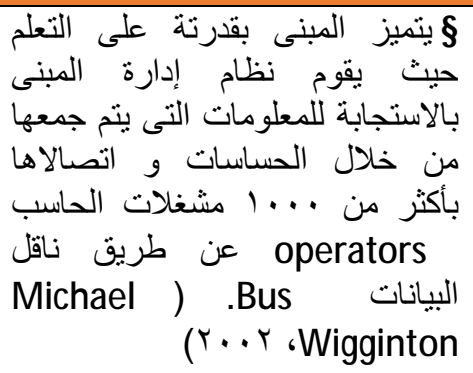 & 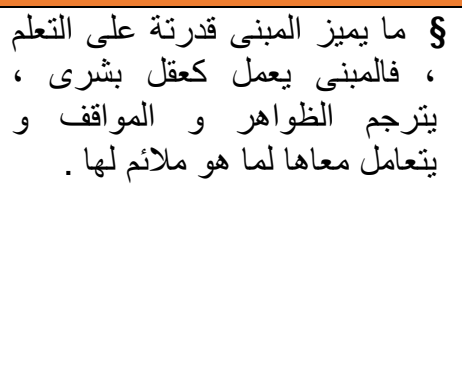 & 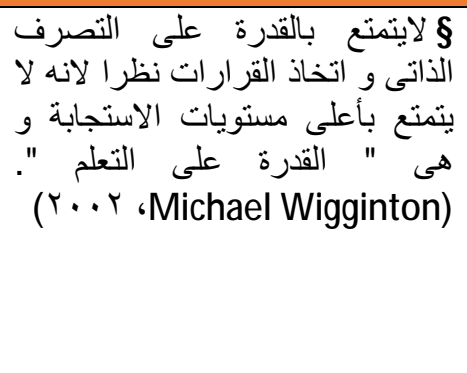 & \\
\hline 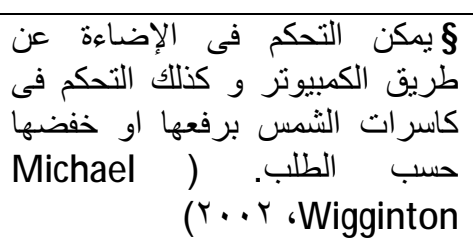 & 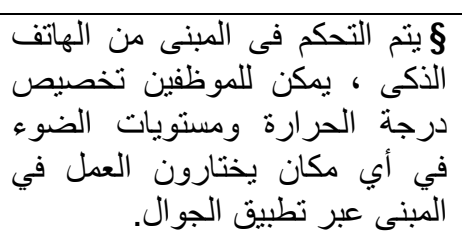 & 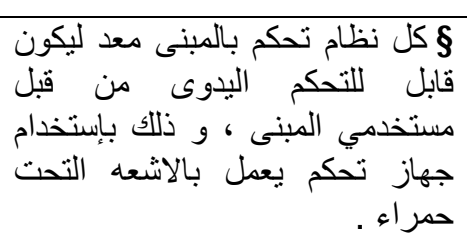 & \\
\hline \multicolumn{4}{|c|}{ التوافق مع البيئة و الاستدامة } \\
\hline 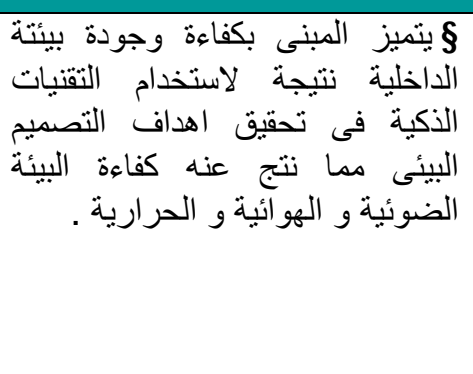 & 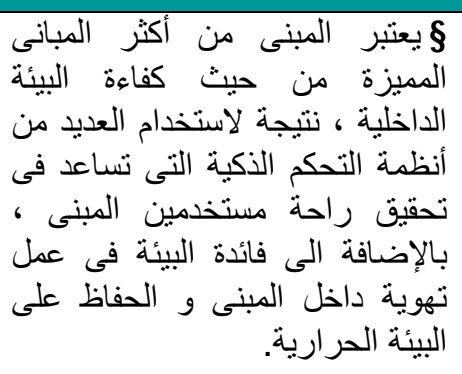 & 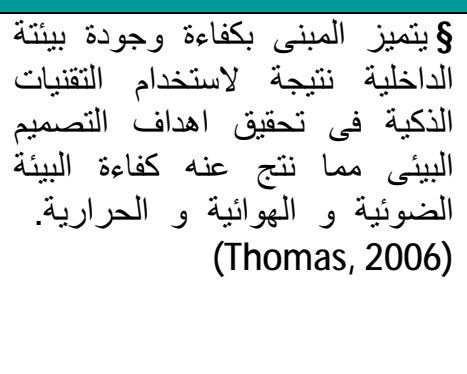 & 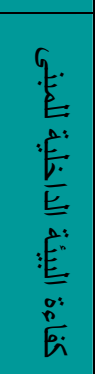 \\
\hline 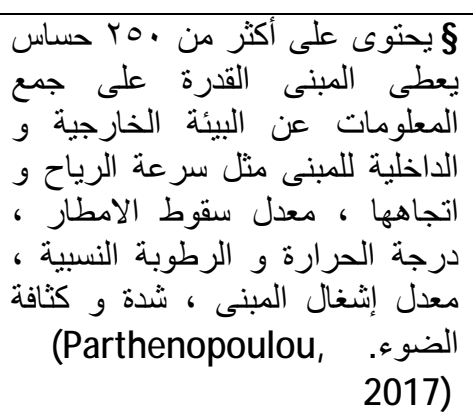 & 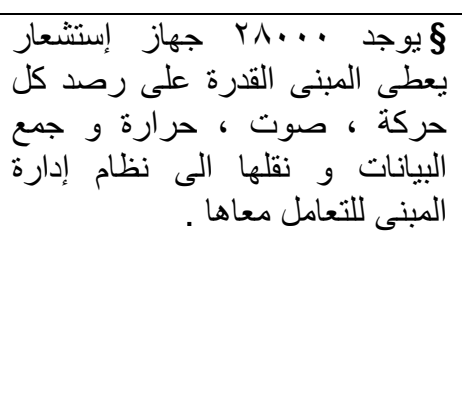 & 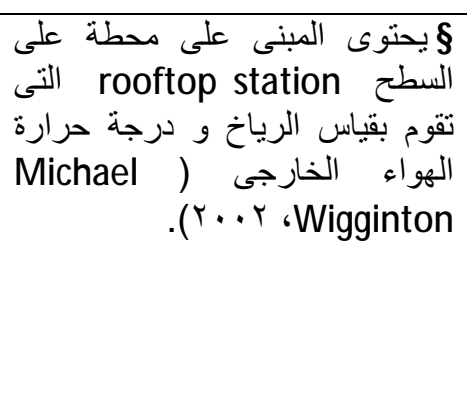 & \\
\hline
\end{tabular}




\begin{tabular}{|c|c|c|c|}
\hline 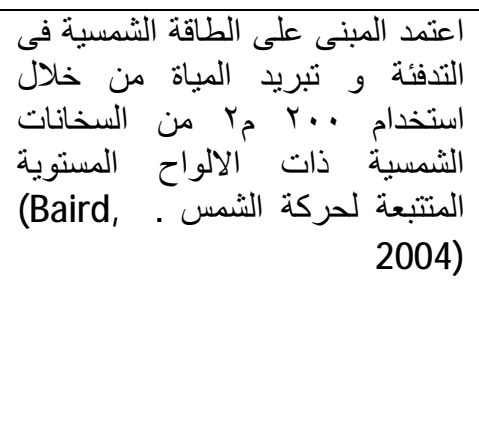 & 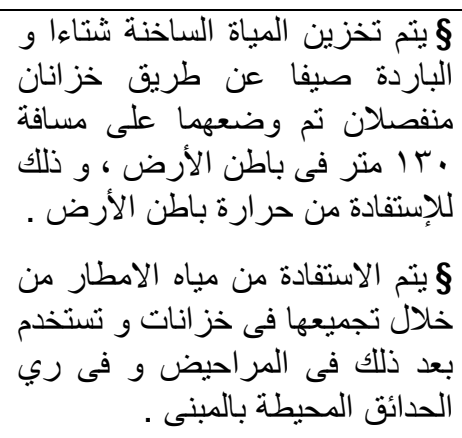 & 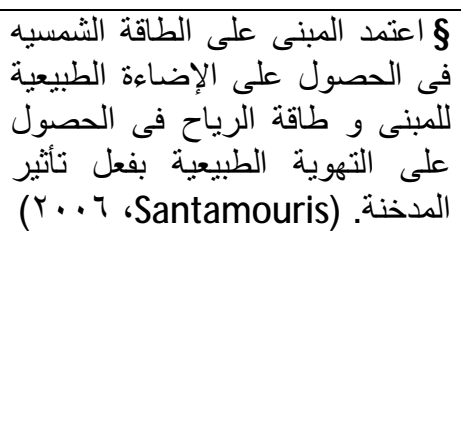 & 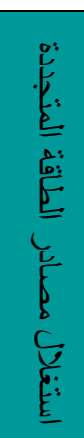 \\
\hline 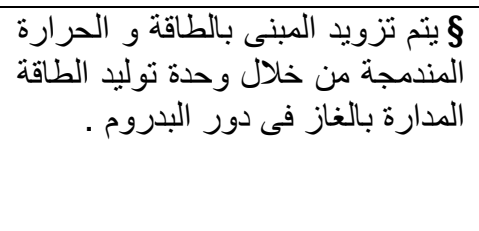 & 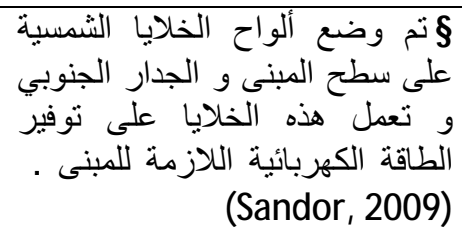 & 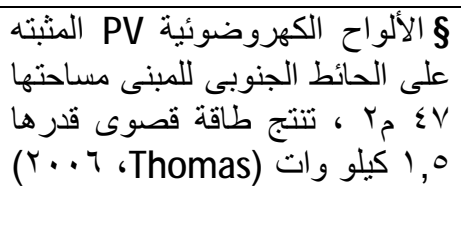 & E: \\
\hline Headquarters of Gotz & $\begin{array}{l}\text { مبنى الحافةة } \\
\text { The edge }\end{array}$ & $\begin{array}{c}\text { المبنى البيئى } \\
\text { The Environmental } \\
\text { Building (BRE) }\end{array}$ & $\begin{array}{l}\text { E: } \\
\underline{5}: \cdot 6\end{array}$ \\
\hline الحر ارية - بيئة التهوية الضائية - البيئة الضية & 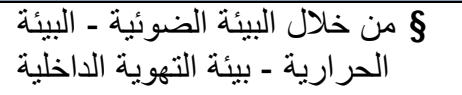 & 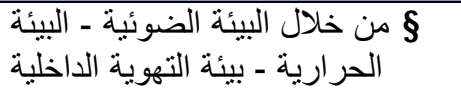 & \\
\hline 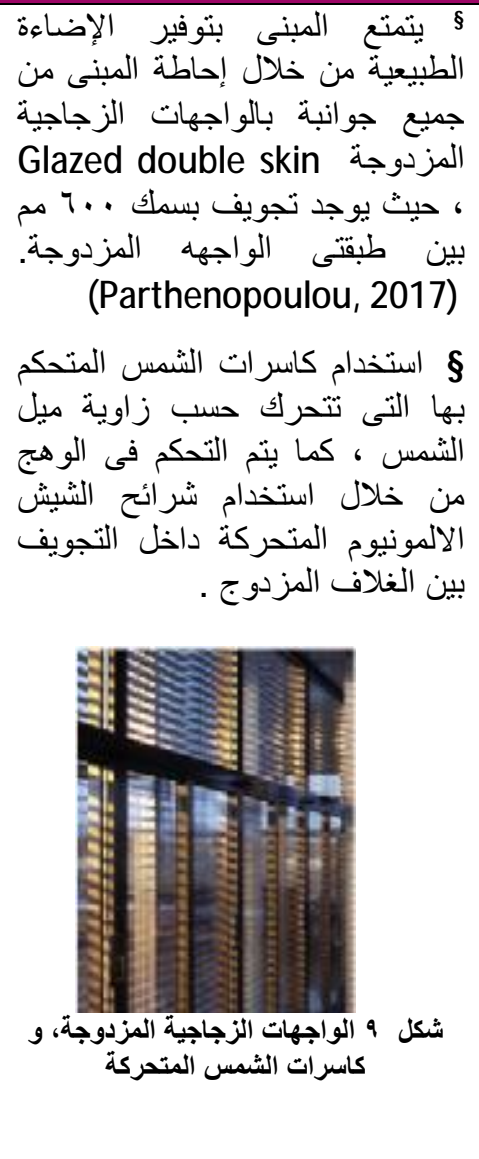 & 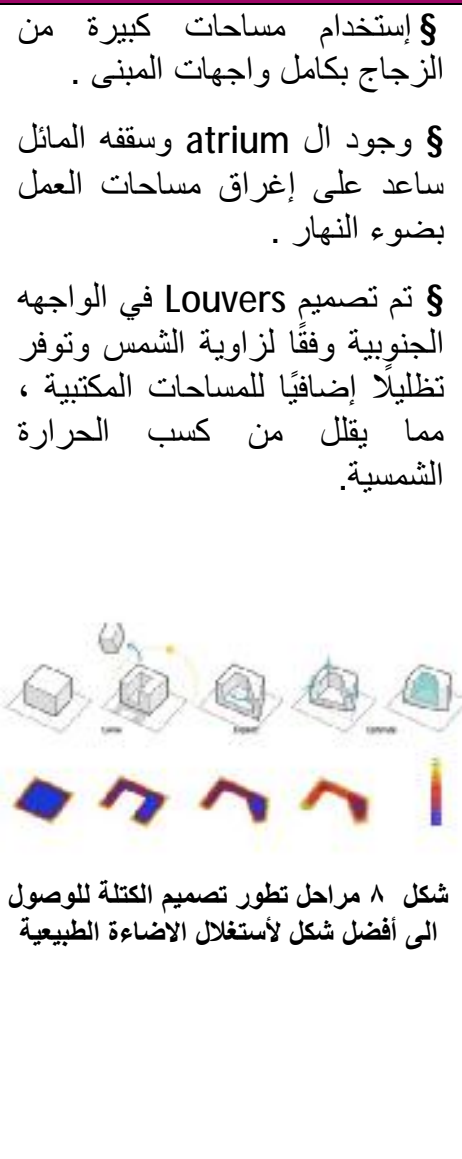 & 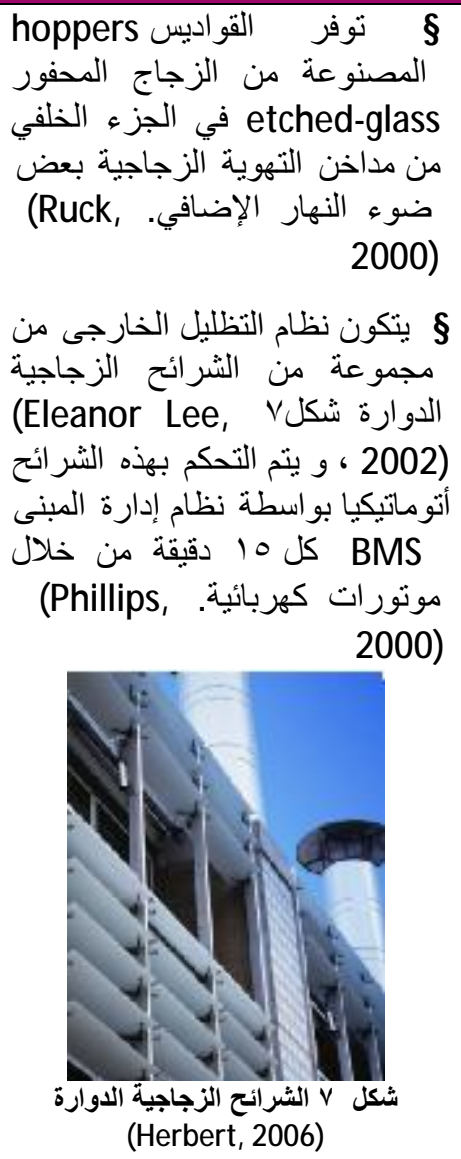 & 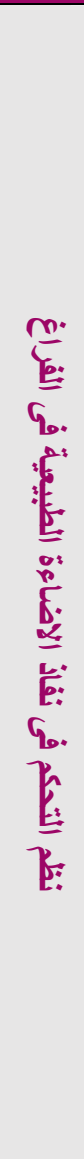 \\
\hline
\end{tabular}




\begin{tabular}{|c|c|c|c|}
\hline 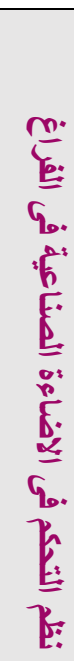 & 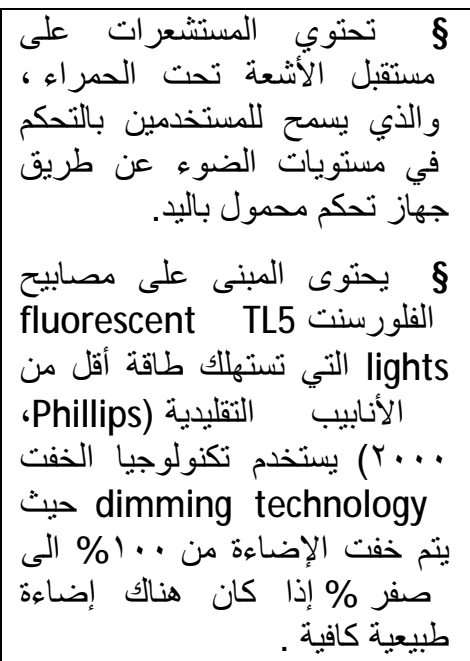 & 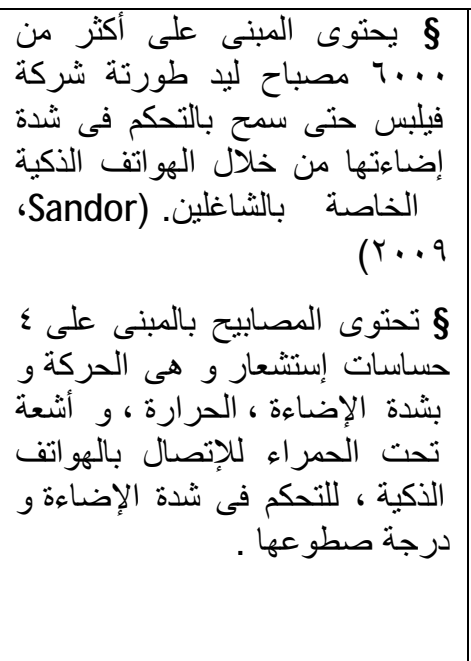 & 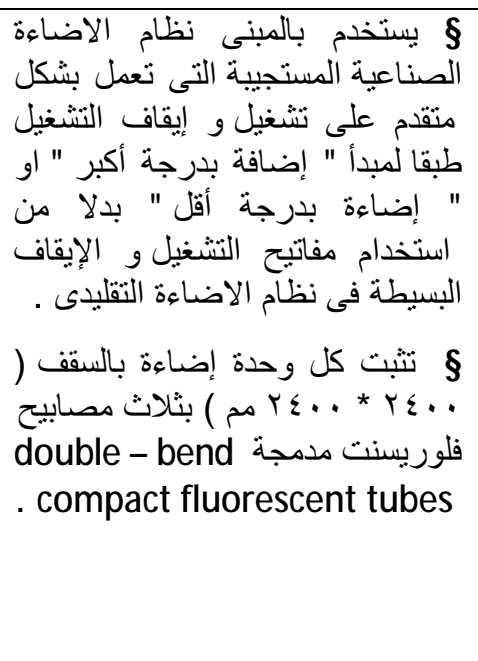 \\
\hline 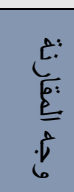 & $\begin{array}{l}\text { The Environmental Building } \\
\text { (BRE) }\end{array}$ & مبنى الحافة & $\begin{array}{c}\text { مبنى شركة جوتز } \\
\text { Headquarters of Gotz }\end{array}$ \\
\hline \multicolumn{4}{|c|}{ التحمث فى البيئة الحرارية داخل المبنى } \\
\hline & 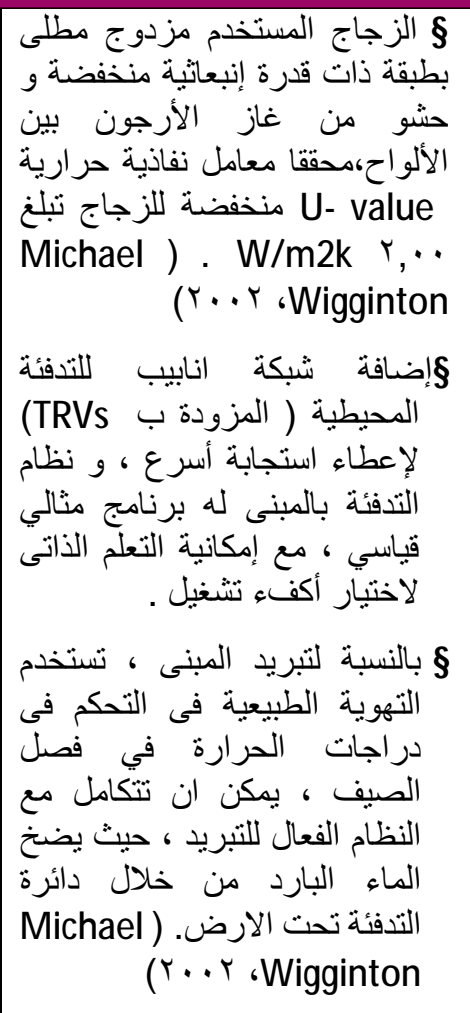 & 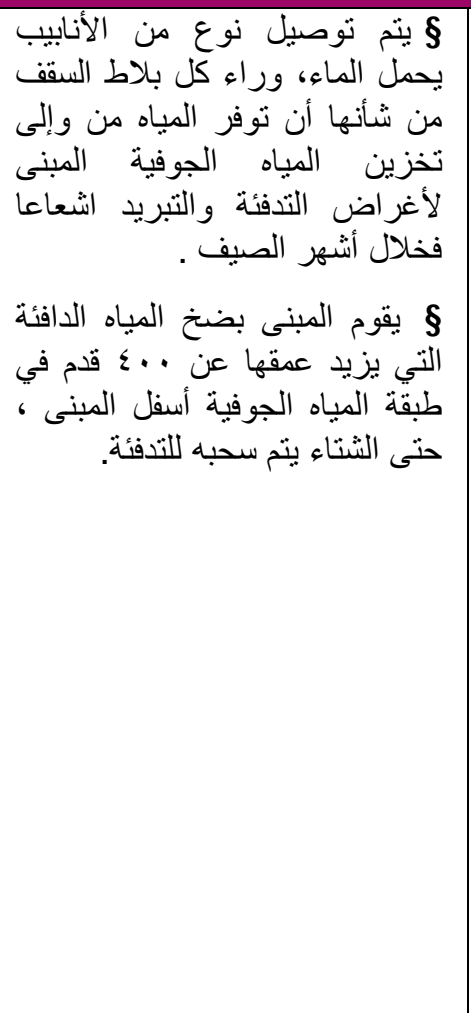 & 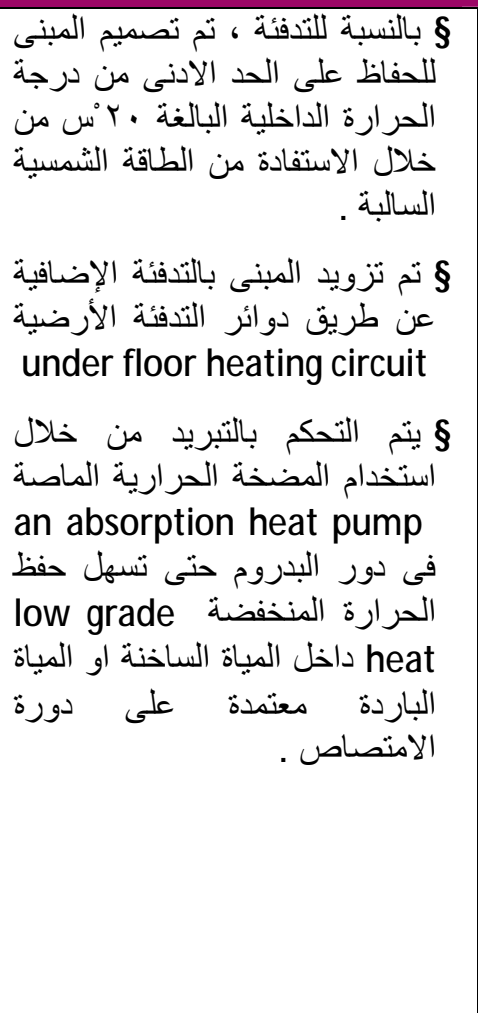 \\
\hline \multicolumn{4}{|c|}{ التحكم فى التهوية داخل المبنى } \\
\hline & 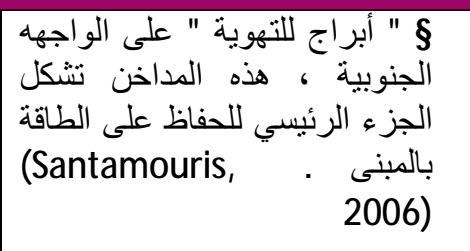 & 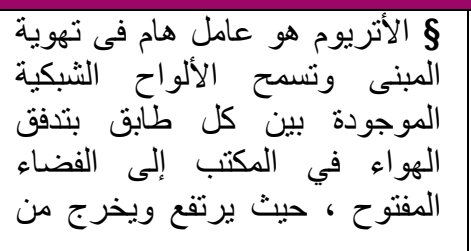 & 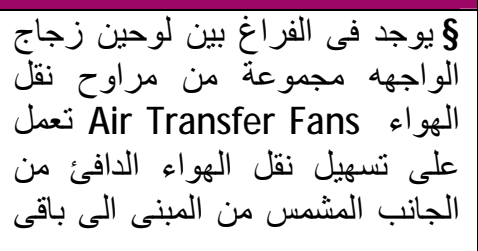 \\
\hline
\end{tabular}




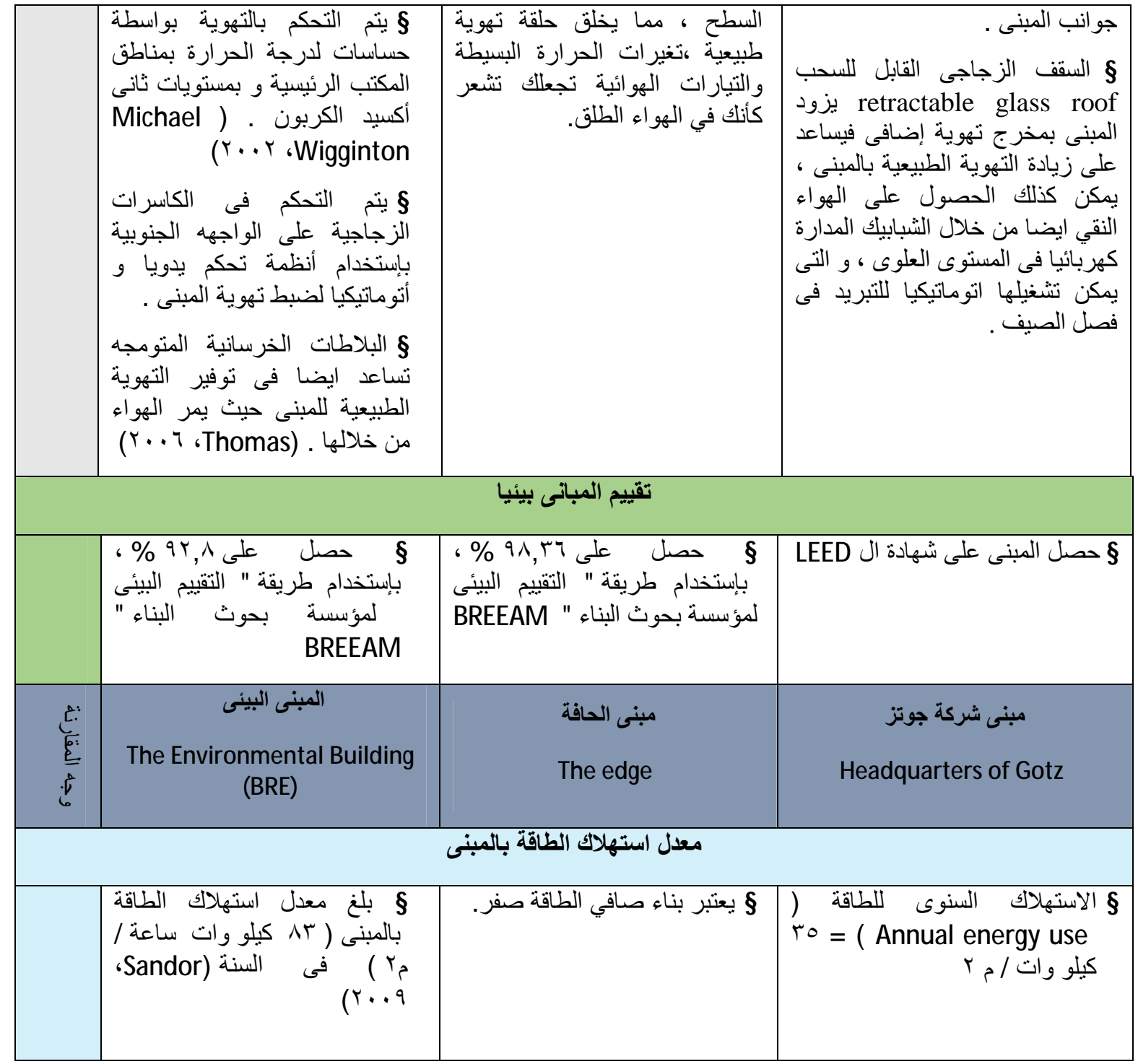

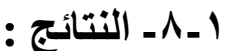

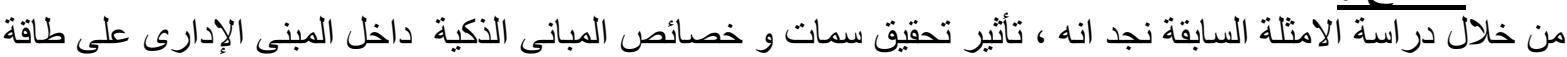

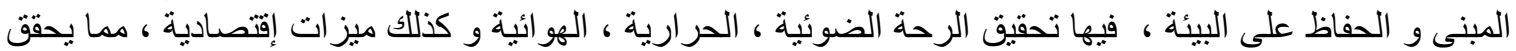

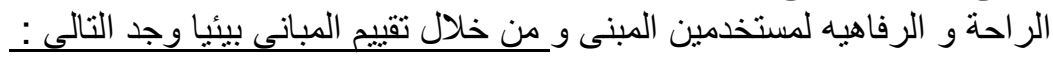

بلغ معدل استهلاك الطاقة بالمبنى البيئي(BRE) The Environmental Building (

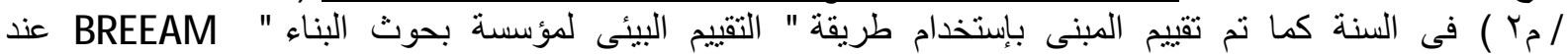

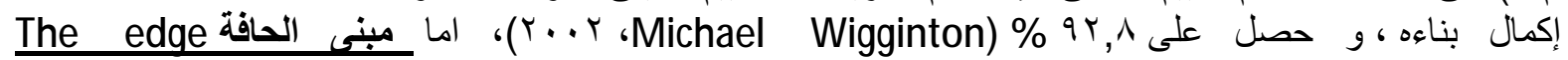

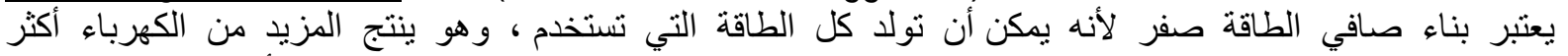

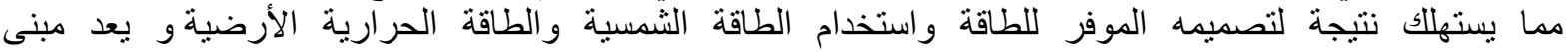

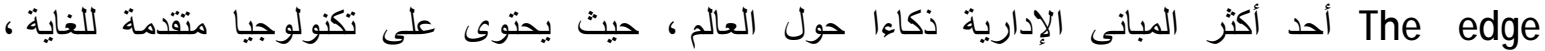

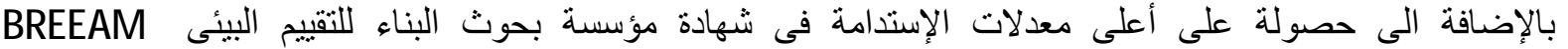

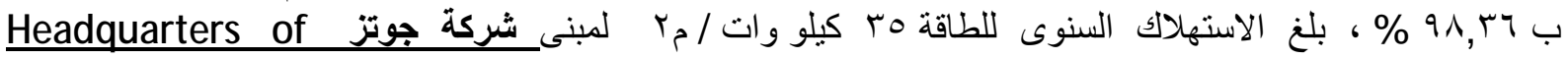

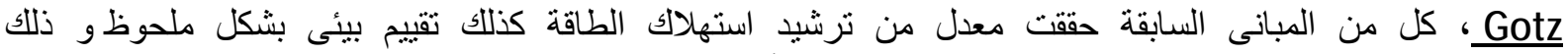
من خلال تطبيق تقنيات المبانى الذكية ، مما يؤكد على الهية أستخدام هذه التقنيات فى المبانى الإدارية . 

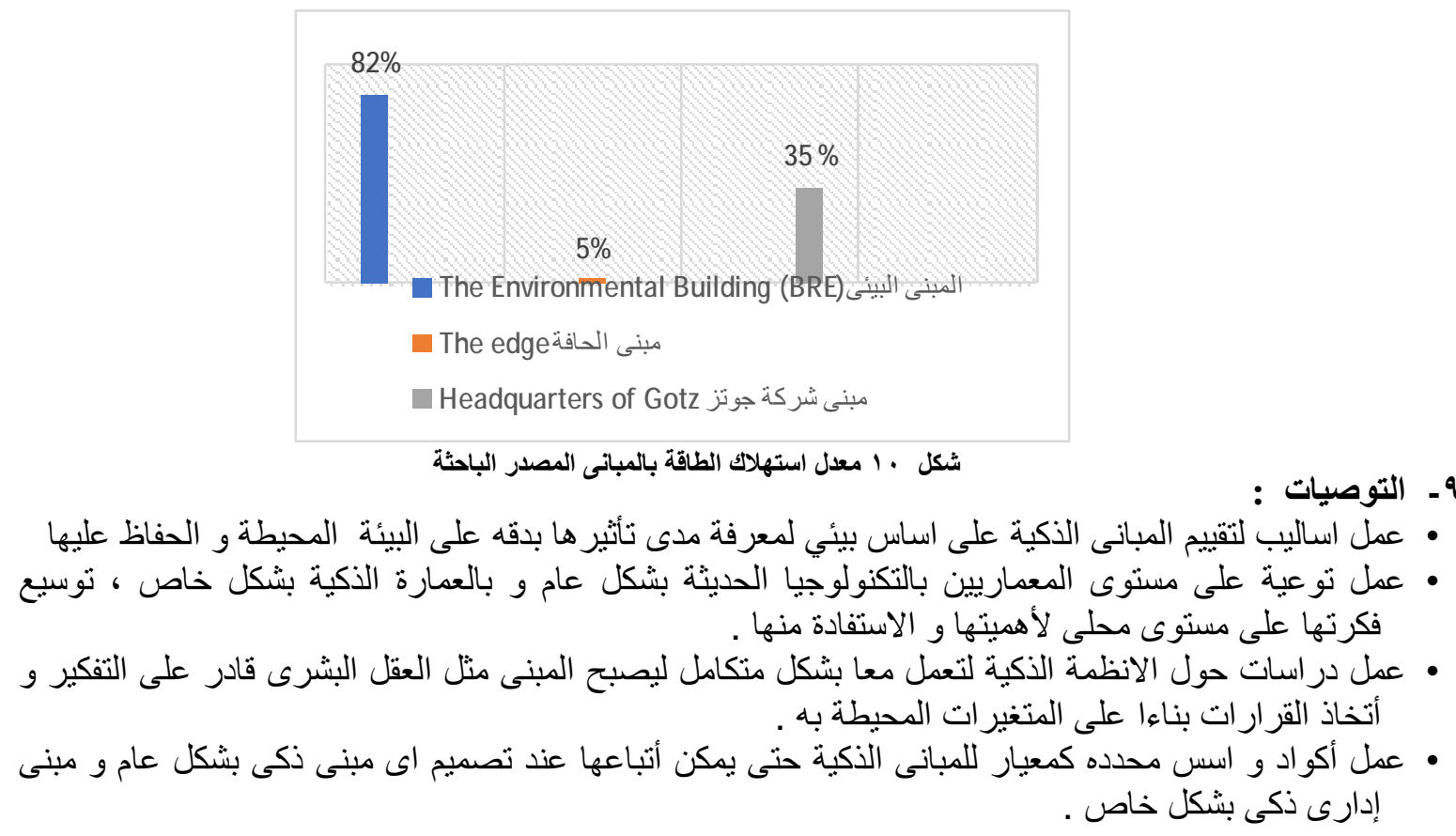

1. Ahmed Reda, M. B. (2010). Intelligent Architecture as an approach for apply the Technological Development in Attaining the Objectives of Sustainable Architecture. Sustainable Architecture BUE. P6.

2. Andrew Harrison, E. L. (2005). Intelligent buildings in south East Asia. Taylor \& Francis; 1 edition. P2.

3. Atkin, B. (1993). Intelligent buildings : applications of IT and building automation to high technology construction projects .p120.

4. Baird, G. (2004). The architectural expression of environmental control systems. London: Taylor \& Francis e-Library. P85.

5. Chuang Wang, D. Y. (2016). Modeling individual's light switching behavior to understand lighting energy use of office building. Energy Procedia.p781.

6. Elazm, F. (2017). Towards Novel and Appropriate Smart Buildings " Beijing Water Cube ". 2 Smart Architecture. International Journal of Environmental Science.

7. Eleanor Lee, S. S. (2002). High-Performance Commercial Building Façades. The Regents of the University of California.p100.

8. Gyeong Yun, K. C. (2014). The influence of shading control strategies on the visual comfort and energy demand of office buildings. Energy Build.p70.

9. Herbert, P. (2006). The environmental building. Structural Survey.

10. Himanen, M. (2003). The intelligence of intelligent buildings : the feasibility of the intelligent building concept in office buildings. Espoo.

11. Kleibrink, M. (2013). smart working smart buildings and the future of work. Light life. P31.

12. Michael Wigginton, J. H. (2002). Intelligent Skins. Architectural Press. P77,78,80,85,96,97.

13. Montbonnot Saint Martin, J. M. (1999). SmartOffice: An Intelligent and Interactive Environment.P104.

14. Parthenopoulou, N. (2017). Sustainability through Intelligence in Buildings. International Journal of Engineering Research and Applications. P17,18.

15. Phillips, D. (2000). Lighting modern buildings. Oxford: Architectural Press. p126,127. 
16. Ruck, N. (2000). Daylight in Buildings - a source book on daylighting systems and components. International Energy Agency.

17. Sandor, M. (2009). The Edge. The Hopkins Review. P7.

18. Santamouris, M. (2006). Enviromental Design of Urban Buildings: An integrated approach. P57,157 .

19. Sinopoli, J. (2006). Smart Buildings. Spicewood Publishing. p12, p7.

20. Sisk, J. ,. (2001). BRE Environmental Building. European Green Building Forum.

21. Sotiris Papantoniou,S.M.(2017).Using Intelligent Building Energy Management System for the Integration of Several Systems to one Overall Monitoring and Management System.Energy Procedia.P 641.

22. Thomas,R.(2006). Environmental Design.An introduction for Architects and Engineers. Taylor \& Francis.

23. Yang, C. (2018). Smart Building Energy Systems. In R. Wang, Handbook of Energy Systems in Green Buildings. P4. 